Original Paper http://ajol.info/index.php/ijbcs http://indexmedicus.afro.who.int

\title{
Influence de quelques paramètres climatiques sur les effectifs de Bemisia tabaci sur la tomate (Solanum lycopersicum L.)
}

\author{
Jean-Claude N'ZII ${ }^{1,2^{*}}$, Christophe KOUAME ${ }^{2}$ et Assanvo Simon-Pierre N'GUETTA ${ }^{1}$ \\ ${ }^{1}$ Laboratoire de Génétique, Unité de Formation et Recherche Biosciences, Université Félix Houphouët-Boigny, \\ 22 BP 582 Abidjan 22, Côte d'Ivoire. \\ ${ }^{2}$ ICRAF-The World Agroforestry Centre, 08 BP 2823 Abidjan 08, Côte d'Ivoire. \\ "Auteur correspondant; E-mail : nzi.jeanclaude@univ-fhb.edu.ci
}

\section{REMERCIEMENTS}

Nous sommes reconnaissants envers le CNRA (Centre National de Recherche Agronomique) pour avoir financé cette étude.

\section{RESUME}

Pour contribuer à la lutte contre la maladie de l'enroulement jaunissant des feuilles de la tomate, un programme de recherche a été initié en Côte d'Ivoire. L'influence des paramètres climatiques sur l'évolution des populations du vecteur de cette maladie virale, la mouche blanche (Bemisia tabaci Genn.) a été évaluée à la Station de Recherche sur les Cultures Vivrières du Centre National de Recherche Agronomique à Bouaké à travers six différentes dates de semis de décembre 2000 à février 2002. Cinq variétés de tomate dont un témoin local ont été testées selon un dispositif en blocs de Fisher répété quatre fois. L'analyse de variance, l'analyse en composantes principales, la corrélation linéaire de Pearson et la régression linéaire ont permis d'analyser les données collectées. Les résultats ont montré une significative influence des trois paramètres climatiques sur les populations de l'insecte. Le modèle linéaire double constitué du couple température moyenne et pluviométrie et du nombre d'adultes et de larves, a été celui qui a mieux décrit les fluctuations des adultes et des larves. Ce modèle a en effet expliqué $35,80 \%$ des fluctuations des populations adultes et $43,20 \%$ de celles des larves montrant ainsi l'influence du climat sur l'insecte.

(c) 2019 International Formulae Group. All rights reserved.

Mots clés : Mouche blanche, tomate, variété, date de semis, enroulement jaunissant des feuilles, Côte d'Ivoire.

\section{Influence of some climatic parameters on the numbers of Bemisia tabaci on tomato (Solanum lycopersicum L.)}

\begin{abstract}
To contribute to the fight against the Tomato yellow leaf curl disease of tomato, a research program has been initiated in Côte d'Ivoire. The influence of climatic parameters on the evolution of populations of the vector of this viral disease, the whitefly (Bemisia tabaci Genn.) was evaluated at the Food Crops Research Station of the National Agronomic Research Center in Bouaké through six different sowing dates from December 2000 to February 2002. Five varieties of tomato, including a local control, were tested in a Fisher block design repeated four times. Analysis of variance, principal component analysis, Pearson linear
\end{abstract}


correlation, and linear regression were applied to the collected data. The results showed a significant influence of the three climatic parameters on the populations of the insect. The linear double model of the mean temperature and rainfall pair and the number of adults and larvae best described the fluctuations in adults and larvae. This model explained $35.80 \%$ of fluctuations in adult populations and $43.20 \%$ of those of larvae, thus showing the influence of climate on whitefly in Côte d'Ivoire.

(C) 2019 International Formulae Group. All rights reserved.

Keywords: Whitefly, tomato, variety, sowing date, Tomato yellow leaf curl, Côte d'Ivoire.

\section{INTRODUCTION}

Cultivée pour ses fruits, la tomate (Solanum lycopersicum L.) se classe en deuxième position parmi les légumes les plus consommés au monde après la pomme de terre (Fondio et al., 2013). Sa production mondiale a rapidement augmenté au cours de la dernière décennie, passant de 141,4 millions de tonnes en 2009 à 177 millions en 2016 (FAO, 2018).

La tomate tient en Côte d'Ivoire une place importante parmi les cultures maraîchères et est surtout cultivée pour sa consommation en frais. Malheureusement, la production nationale est encore insuffisante pour satisfaire les besoins de la population ivoirienne estimés à plus de 100000 tonnes/an (Fondio et al., 2013). C'est une culture de saison sèche parce que les meilleurs rendements y sont obtenus. Des travaux ont montré que les facteurs climatiques et édaphiques favorisent une forte pression parasitaire qui limite considérablement la production de la culture (Fondio et al., 2013 ; Camara et al., 2016 ; Babu et al., 2018 ; Didi et al., 2018). A cela, il faut ajouter l'absence de variétés de tomate adaptées et performantes. En effet, les variétés cultivées ou commercialisées ne supportent pas généralement les conditions chaudes et humides du pays qui sont responsables du développement de nombreuses maladies fongiques, bactériennes et virales et de la pullulation des ravageurs (insectes et acariens). Aussi, le développement de la culture de la tomate nécessite-t-il la sélection de variétés adaptées à ces conditions locales. En fait, ces dernières années, les contraintes majeures à la production de la tomate identifiées en Afrique de l'Ouest et particulièrement en Côte d'Ivoire, sont l'apparition en saison sèche, de viroses endémiques (AVRDC, 2005; Gilberston et Shetty 2006 ; N'zi et al., 2010; Camara et al., 2016). Une recrudescence de maladies virales ou maladies émergentes causées par des Geminiviridae dont l'une des plus sévères appelée Enroulement jaunissant des feuilles de la tomate ou Tomato Yellow Leaf Curl Disease (TYLCD), a été observée partout dans le monde tropical et subtropical (N'zi, 2010 ; Camara et al., 2016; Babu et al., 2018). Le virus responsable de la maladie, le Tomato yellow leaf curl virus (TYLCV) selon Rubio et al. (2003) est transmis par la mouche blanche, Bemisia tabaci Gennadius (N'zi et al., 2010). Cette mouche, dont la prolifération en Afrique tropicale est probablement liée aux facteurs climatiques et à l'usage exclusif de pyréthrinoïdes de synthèse qui entraine le phénomène de résistance chez le ravageur, représente à elle seule un double danger (Gamarra et al., 2016) de par sa capacité à engendrer des dégâts directs sur les plantes, à transmettre le TYLCD et d'autres maladies virales sur la patate douce au Burkina-Faso (Koussoube et al., 2018) et sur le cotonnier en Côte d'Ivoire (Didi et al., 2018) et au Sénégal selon Sarr et al. (2016). En effet, le rendement est amoindri car, la fonction photosynthétique 
et la qualité des produits récoltés sont affectées par l'excrétion du miellat (Lida et al., 2009). La prolifération de cet insecte ces dernières années, surtout en 2014 et 2015 tendrait à faire de lui le ravageur dominant sur le cotonnier en Côte d'Ivoire (Didi et al., 2018).

La succession des saisons climatiques au cours de l'année est connue. Cependant, on assiste ces dernières années à des changements climatiques d'une année à l'autre tant dans la durée que dans les effets (Kouassi et al., 2010; Ahoussi et al., 2013). Ce manuscrit traite d'un sujet scientifique d'actualité car avec le changement climatique l'impression d'une recrudescence de ce ravageur dans la sous-région Ouest-africaine est donnée (Didi et al., 2018; Koussoube et al., 2018). La recherche de variétés tolérantes à cette maladie, à son vecteur et productives paraît la solution indiquée pour aider les producteurs à améliorer la productivité de cette culture (Babu et al., 2018). C'est dans cette optique que cette étude est proposée en vue d'identifier le comportement de l'insecte vecteur sur la tomate en fonction de la date de semis, et d'actualiser les données bioclimatiques pour la lutte contre la mouche blanche. Spécifiquement, cette étude avait pour but de déterminer l'influence de quelques paramètres climatiques sur l'évolution des effectifs de $B$. tabaci dans les champs de tomate à Bouaké en Côte d'Ivoire.

\section{MATERIEL ET METHODES}

\section{Site d'étude}

Cette étude a été réalisée à la Station de Recherche sur les Cultures Vivrières (SRCV) du Centre National de Recherche Agronomique (CNRA) à Bouaké, ville située au Centre de la Côte d'Ivoire entre les méridiens $5^{\circ} 7^{\prime} 00^{\prime \prime e t}$ $4^{\circ} 58^{\prime} 00^{\prime \prime}$ de longitude Ouest et les parallèles $7^{\circ} 45^{\prime} 00^{\prime \prime}$ et $7^{\circ} 38^{\prime} 00^{\prime \prime}$ de latitude Nord à une altitude de $376 \mathrm{~m}$ et à environ $370 \mathrm{~km}$ par la route de la capitale économique Abidjan. La pluviométrie moyenne annuelle est comprise entre 1100 et $1200 \mathrm{~mm}$ (Fondio, 2005). Les sols de la Station sont ferralitiques, gravillonnaires, remaniés, peu profonds et issus d'un matériau d'altération granitique (N'gbesso et al., 2013). L'analyse au Laboratoire Central Sol-Plante-Eau du CNRA à Bouaké d'échantillons de sol prélevés dans les parcelles avant la mise en place de l'étude, a révélé que le sol a une texture sableuse faiblement argilo limoneuse avec 60\% de sable, $20 \%$ d'argile et $20 \%$ de limon (Fondio, 2005).

\section{Matériels}

\section{Matériel végétal}

Le matériel végétal était constitué de cinq variétés de tomate dont un témoin local (ABBA), deux lignées issues de la collection du CNRA (IDSA108 et IDSA109) et deux variétés de l'AVRDC-The World Vegetable Center reconnues tolérantes au TYLCV (CLN2116B et CLN2123A). Ces cinq variétés ont été évaluées à la Station de Recherche sur les Cultures Vivrières du CNRA à Bouaké.

\section{Méthodes}

Les expérimentations pour déterminer l'évolution de la mouche blanche à six différentes dates de semis d'environ cinq mois chacune ont été réalisées les 05 Décembre 2000, 12 mars 2001, 08 juin 2001, 10 Août 2001, 22 octobre 2001 et 02 février 2002.

\section{Dispositif expérimental}

A chaque date de semis, l'essai était disposé en blocs de Fisher avec quatre répétitions. La parcelle élémentaire était de $5 \mathrm{x}$ $2 \mathrm{~m}$ et la superficie de la parcelle utile a été de 5 $\mathrm{m}^{2}$. Chaque variété de tomate était représentée par deux lignes de 12 plants avec $50 \mathrm{~cm}$ entre les lignes et $40 \mathrm{~cm}$ entre les plants de la même 
ligne, soit 24 plants par parcelle donnant ainsi une population de 24000 plants à l'hectare.

\section{Pratiques culturales}

Une pépinière a été réalisée lors de chaque essai sur des planches aménagées à cet effet. Une planche de pépinière de $1 \mathrm{~m}$ de large et $2 \mathrm{~m}$ de long a donc été réalisée. Deux ou trois grains ont été semés dans des poquets distants de $5 \mathrm{~cm}$ sur la ligne avec $10 \mathrm{~cm}$ entre les lignes. Des binages fréquents ont été réalisés pour entretenir la pépinière afin d'éviter que le sol ne se tasse et durcisse. Vingt et un jours après le semis, les plants de la pépinière ont été repiqués sur billon selon le dispositif mentionné ci-dessus. Pour ce faire, une parcelle a été labourée pour confectionner les billons de $5 \mathrm{~m}^{2}(5 \times 1 \mathrm{~m})$ auxquels une fumure de fond (NPK 10-18-18) à raison de 300 $\mathrm{kg} / \mathrm{ha}$ a été apportée. Ensuite, deux applications d'urée (100 kg/ha) et de potasse ( $200 \mathrm{~kg} / \mathrm{ha})$ ont été effectuées à la deuxième et cinquième semaine après le repiquage. Une irrigation gravitaire régulière et des sarclages manuels réalisés pour contrôler l'enherbement ont été faits à la demande. Aucun traitement phytosanitaire n'a été effectué pour préserver les insectes notamment les populations de la mouche blanche.

\section{Paramètres mesurés}

Mesures des paramètres climatiques

Les données climatiques utilisées pour les analyses ont été fournies par la Société d'Exploitation Aéroportuaire et Météorologique (SO.D.EX.A.M) qui fait des prélèvements tous les 10 jours. Trois paramètres climatiques ont été analysés, et leurs effets sur l'évolution des effectifs de la mouche blanche sur la tomate ont été évalués. Il s'agit de: la température moyenne (TMOY), la pluviométrie (PLUIE) et l'humidité relative (HMOY). Pour collecter ces données météorologiques, des thermomètres à alcool ou à mercure ont été utilisés pour relever la température, l'hygromètre mécanique à ressort mécanique pour mesurer l'humidité relative et le pluviomètre manuel avec bague de cumul pour mesurer la pluviométrie tous les 10 jours. Mesure des paramètres des populations de B. tabaci

Le nombre d'adultes de mouche blanche (NBAD) a été dénombré par semaine à partir du $35^{\mathrm{e}}$ jusqu'au $84^{\mathrm{e}}$ jour après le semis (JAS), sur toutes les feuilles d'un échantillon de dix plants par parcelle, choisis au hasard. Le nombre de larves de mouche blanche (NBLAV) situées sur la face inférieure des feuilles de tomate a été compté par semaine à partir du $35^{\mathrm{e}}$ jusqu'au $84^{\mathrm{e}}$ jour après le semis (JAS) à l'aide d'une loupe binoculaire sur une feuille prélevée au hasard pour un total de dix plants par parcelle.

\section{Méthodes d'analyse des données}

Les analyses statistiques basées sur la statistique descriptive (moyenne, écart-type, coefficient de variation et coefficient de corrélation) de chacune des variables a été effectuée. Une analyse de variance en utilisant le modèle linéaire généralisé du logiciel SAS (SAS, 2003) a été réalisée pour déceler s'il y a une différence significative ou non entre les traitements testés au cours de l'expérimentation pour un paramètre mesuré ou observé. Pour les variables significatives, le test de Duncan pour a été utilisé pour séparer les moyennes par groupes homogènes au seuil de 5\%. Par ailleurs, une analyse en Composantes Principales (ACP) à l'aide du logiciel XLSTAT 7.5.3 pour regrouper ou distinguer un ensemble d'unités en fonction d'un ensemble de variables. La régression a permis de modéliser les fluctuations des populations de l'insecte vecteur en fonction des paramètres climatiques. Le poids de la variable explicatrice qu'est le climat sur la variable expliquée constituée par les populations de la mouche blanche a été mesuré à travers le coefficient de détermination $\mathrm{R}^{2}$ avec le logiciel XLSTAT 7.5.3. 


\section{RESULTATS}

\section{Statistiques élémentaires et examen de la variabilité climatique}

L'analyse des résultats provenant des statistiques élémentaires obtenus au niveau des trois paramètres climatiques de la zone de Bouaké par date de semis a indiqué que le semis réalisé en février 2002 a enregistré la plus grande pluviométrie avec $59,5 \mathrm{~mm}$ et celui d'octobre 2001 la plus petite avec $9,2 \mathrm{~mm}$ de pluie. Les semis de décembre 2000 avec 28,1 ${ }^{\circ} \mathrm{C}$ et de février 2002 avec $27,6{ }^{\circ} \mathrm{C}$ ont été les périodes les plus chaudes, contrairement aux semis de juin 2001 et d'août 2001 qui ont enregistré $24,1{ }^{\circ} \mathrm{C}$ et $25,1{ }^{\circ} \mathrm{C}$, respectivement. En ce qui concerne l'humidité relative, le semis de juin 2001 a été considéré comme le plus humide avec $86 \%$. Le semis de décembre 2000 a enregistré l'humidité relative la moins élevée avec 56\%, suivi de celui d'octobre 2001 avec $67 \%$ (Tableau 1).

L'examen de la variabilité des paramètres climatiques par date de semis à travers le coefficient de variation a généré une variation de $60,93 \%$ pour le semis d'août 2001 à $219,43 \%$ pour celui de décembre 2000 en ce qui concerne la pluviométrie. La température moyenne a très peu varié car le taux de variation a oscillé entre $1,21 \%$ pour le semis d'octobre 2001 et 3,99\% pour celui d'août 2001. Quant à la variation de l'humidité moyenne, elle a fluctué entre $1,90 \%$ pour le semis de mars 2001 et $16,45 \%$ pour celui de décembre 2000 . En conclusion, la pluviométrie a été le paramètre climatique qui a enregistré la plus grande dispersion de ses moyennes par semis.

\section{Relation entre les 3 paramètres climatiques}

Les coefficients de corrélation de Pearson ont indiqué dans le tableau 2 que l'humidité relative est en relation avec les deux autres paramètres climatiques que sont la pluviométrie et la température moyenne à tous les semis, excepté le semis de juin 2001. Seule une relation a été observée entre la pluviométrie et la température moyenne $(\mathrm{r}=$ 0,77) au semis d'août 2001. Autrement dit, la connaissance de la valeur de l'une ou l'autre de ces variables peut permettre d'estimer dans une certaine mesure celle de l'autre variable à cette période.

\section{Contribution des paramètres climatiques à la} variation des populations de $B$. tabaci

Identification du modèle linéaire associé aux paramètres climatiques et aux populations de B. tabaci adultes par plant

Dans l'ensemble, le meilleur modèle linéaire simple décrivant les variations de la population adultes de la mouche blanche est formé par le nombre d'adultes et la température moyenne (Tableau 3). L'effet de la température moyenne a varié de manière significative $(P<0,0001)$. La part de la variation des populations de la mouche blanche expliquée par la température moyenne est de $30,80 \%$. Cette variable a contribué à la construction de la droite de régression simple dont l'équation associée est: $\mathrm{NBAD}=$ $16,2627+0,7503 \times$ TMOY.

Cette équation a révélé que l'effectif d'adultes de l'insecte augmente en moyenne de 0,75 quand les températures moyennes augmentent de $1{ }^{\circ} \mathrm{C}$. En plus, cet effectif serait de $-16,26$ adultes si les températures moyennes étaient nulles.

Le meilleur modèle linéaire double est celui constitué du nombre d'adultes et du couple température moyenne et pluviométrie. En effet, ce modèle a expliqué $35,80 \%$ des fluctuations des populations d'adultes de la mouche blanche. Les effets de la température moyenne et de la pluviométrie ont significativement varié $(\mathrm{P} / \mathrm{TMOY}=0,0001<$ $0,05 ;$ P/PLUIE $=0,04<0,05)$. Par conséquent, les températures moyennes et la pluviométrie ont contribué à la construction de la droite de régression double dont l'équation associée est : NBAD $=-16,1129+$ $0,7608 \times$ TMOY $-0,0150 \times$ PLUIE. 
Cette équation indique que l'effectif d'adultes de la mouche blanche diminue en moyenne de 0,015 adulte lorsque la pluviométrie augmente en moyenne de $1 \mathrm{~mm}$. Cette même variable augmente de 0,76 adulte quand la température moyenne augmente de 1 ${ }^{\circ} \mathrm{C}$. Cet effectif serait de - 16,11 adultes si les températures moyennes et la pluviométrie étaient nulles c'est-à-dire quand il ne pleut pas et que les températures chutent à $0{ }^{\circ} \mathrm{C}$.

Le meilleur modèle linéaire triple n'a pas mieux décrit les variations de la population des adultes de la mouche blanche que celui à deux paramètres climatiques. Les effets de l'humidité relative n'ont pas été significativement différents $(\mathrm{P}=0,456>$ $0,05)$. Le modèle linéaire à trois paramètres climatiques se résume par conséquent à celui à deux paramètres que sont la pluviométrie et la température moyenne. Le modèle linéaire à deux paramètres représente ainsi le meilleur modèle linéaire de prédiction de la fluctuation des populations d'adultes de la mouche blanche.

\section{Identification du modèle linéaire associé aux paramètres climatiques et aux populations de larves de B. tabaci par feuille}

Le meilleur modèle linéaire simple décrivant les variations de la population de l'insecte est formé par le couple nombre de larves de la mouche blanche et température moyenne (Tableau 4). Ce modèle linéaire a expliqué 30,50\% de la variation totale. L'effet de la température moyenne a significativement varié $(\mathrm{P} / \mathrm{TMOY}=0,0001<$ $0,05)$. La température a ainsi contribué à la formation de l'équation de droite de régression simple: NBLAV $=-15,6109+$ $0,6630 \times$ TMOY .

Cette équation indique que le nombre de larves de l'insecte augmente en moyenne de 0,66 larve quand la température moyenne augmente de $1{ }^{\circ} \mathrm{C}$. Ce même effectif serait de - 15,61 larves par feuille si les valeurs de la température moyenne étaient nulles.
Le meilleur modèle linéaire double qui décrit le mieux la fluctuation des larves est constituée du nombre de larves et du couple (pluviométrie et température moyenne). $\mathrm{Ce}$ modèle a expliqué $43,20 \%$ des fluctuations des populations de larves de la mouche blanche. Les effets de la température moyenne et de la pluviométrie ont significativement varié $(\mathrm{P} / \mathrm{TMOY}=0,0001<0,05 ; \mathrm{P} / \mathrm{PLUIE}=$ $0,003<0,05)$. Par conséquent, la température moyenne et la pluviométrie ont contribué à la construction de la droite de régression double dont l'équation associée est: NBLAV = $15,3972+0,6780 \times$ TMOY $-0,0214 \times$ PLUIE.

Cette équation révèle que l'effectif de larves de la mouche blanche diminue en moyenne de 0,02 larve lorsque la pluviométrie augmente en moyenne de $1 \mathrm{~mm}$. Cette même variable augmente de 0,68 larve quand la température moyenne augmente de $1{ }^{\circ} \mathrm{C}$. Cet effectif serait de $-15,40$ larves par feuille si les températures moyennes et la pluviométrie étaient nulles.

Le meilleur modèle linéaire triple n'a pas mieux décrit les variations de la population des larves par feuille de la mouche blanche comparé à celui à deux paramètres climatiques; l'effet de l'humidité relative n'ayant pas été significatif $(\mathrm{P}=0,134>0,05)$. Le modèle linéaire à trois paramètres climatiques se résume par conséquent à celui à deux paramètres: la pluviométrie et la température moyenne. Il représente le meilleur modèle linéaire de prédiction de la fluctuation des populations de larves de la mouche blanche en fonction des paramètres climatiques.

\section{Influence des paramètres climatiques sur} l'évolution des populations de B. tabaci

Les résultats de l'Analyse en Composantes Principales (ACP) portant sur les cinq variables ont montré que dans l'ensemble, le plan 1-2 a expliqué $77,31 \%$ de la variance. L'axe 1 a expliqué $56,50 \%$ de la 
variation alors que l'axe 2 en a expliqué $20,81 \%$. La structuration globale des trois paramètres climatiques et des deux types de populations de la mouche blanche a engendré trois groupes: le premier groupe, formé par trois variables, le nombre de larves de la mouche blanche, la température moyenne et l'humidité relative a contribué à hauteur de $75,72 \%$ à la construction de l'axe 1, le second constitué par la pluviométrie qui a contribué à $77,93 \%$ à la réalisation de l'axe 2 et le troisième formé uniquement par les populations d'adultes de la mouche blanche qui a contribué à $69,05 \%$ à la construction de l'axe 3 . Cette structuration a montré que la température moyenne, l'humidité relative et la pluviométrie influencent les effectifs de la mouche blanche (Figure 2).

\section{Relation entre les 3 paramètres climatiques et les populations de Bemisia tabaci}

Une corrélation positive et significative $(\mathrm{r}=0,56)$ a été observée entre la température moyenne et les populations d'adultes de la mouche blanche (Tableau 5). Une telle corrélation a été aussi trouvée avec les populations de larves de l'insecte $(\mathrm{r}=$ 0,55). Cela implique que la température influence positivement les populations de la mouche blanche.

Une corrélation négative $(\mathrm{r}=-0,34)$ a été observée entre la pluviométrie et les populations de larves de la mouche blanche. $\mathrm{Ce}$ qui signifie que la pluie influence négativement les populations de la mouche blanche; avec l'accroissement des quantités d'eau tombées, l'effectif des larves de l'insecte diminue dans les champs. Aucune corrélation n'a été observée entre la pluviométrie et les populations d'adultes de la mouche blanche par plant, la pluie n'aurait donc pas d'influence significative sur les effectifs des adultes de l'insecte.

Une corrélation significative et négative $(\mathrm{r}=-0,41)$ a été enregistrée entre l'humidité relative et les populations d'adultes par plant de la mouche blanche. Une telle corrélation a été aussi observée entre les populations de larves de la mouche blanche et ce paramètre climatique $(\mathrm{r}=-0,69)$. Toute augmentation de l'humidité réduirait les effectifs des adultes et des larves de l'insecte.

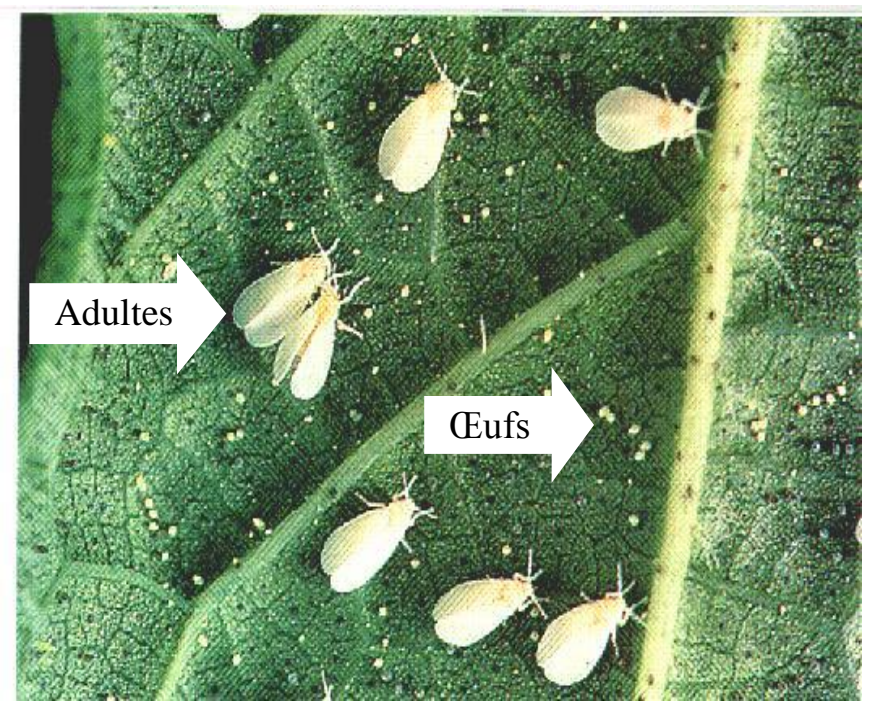

Figure 1 : Adultes et œufs de B. tabaci sur la face inférieure d'une feuille en station (N'zi, 2010). 
Tableau 1 : Statistiques élémentaires des paramètres climatiques par date de semis.

\begin{tabular}{|c|c|c|c|c|c|c|}
\hline $\begin{array}{l}\text { Dates de } \\
\text { semis }\end{array}$ & Variables & Minimum & Maximum & Moyenne & $\begin{array}{l}\text { Ecart- } \\
\text { type }\end{array}$ & $\begin{array}{l}\mathrm{CV} \\
(\%)\end{array}$ \\
\hline \multirow{3}{*}{ Déc. 2000} & Pluviométrie (mm) & 0,000 & 78,000 & 12,575 & 27,593 & 219,43 \\
\hline & Température $\left({ }^{\circ} \mathrm{C}\right)$ & 27,200 & 28,700 & 28,150 & 0,532 & 1,89 \\
\hline & Humidité relative (\%) & 46,000 & 70,000 & 56,125 & 9,234 & 16,45 \\
\hline \multirow{4}{*}{$\begin{array}{l}\text { Mar. } \\
2001\end{array}$} & Pluviométrie (mm) & 5,600 & 82,600 & 42,838 & 28,293 & 66,05 \\
\hline & & & & & & 2,55 \\
\hline & Température $\left({ }^{\circ} \mathrm{C}\right)$ & 25,300 & 27,200 & 26,438 & 0,674 & \\
\hline & Humidité relative (\%) & 77,000 & 82,000 & 79,500 & 1,512 & 1,90 \\
\hline \multirow{3}{*}{ Juin 2001} & Pluie (mm) & 0,200 & 73,900 & 22,338 & 24,450 & 109,45 \\
\hline & Température $\left({ }^{\circ} \mathrm{C}\right)$ & 23,600 & 24,800 & 24,125 & 0,427 & 1,77 \\
\hline & Humidité relative (\%) & 83,000 & 92,000 & 86,750 & 2,712 & 3,13 \\
\hline \multirow{4}{*}{$\begin{array}{l}\text { Août } \\
2001\end{array}$} & Pluviométrie (mm) & 7,100 & 48,100 & 23,975 & 14,608 & 60,93 \\
\hline & & & & & & 3,99 \\
\hline & Température $\left({ }^{\circ} \mathrm{C}\right)$ & 23,600 & 26,200 & 25,125 & 1,002 & \\
\hline & Humidité relative (\%) & 73,000 & 85,000 & 79,625 & 4,274 & 5,37 \\
\hline \multirow{3}{*}{ Oct. 2001} & Pluviométrie (mm) & 0,000 & 29,900 & 9,225 & 11,944 & 129,67 \\
\hline & Température $\left({ }^{\circ} \mathrm{C}\right)$ & 26,000 & 26,900 & 26,388 & 0,318 & 1,21 \\
\hline & Humidité relative (\%) & 49,000 & 75,000 & 67,125 & 10,934 & 16,20 \\
\hline \multirow{3}{*}{ Fév. 2002} & Pluviométrie (mm) & 14,100 & 114,900 & 59,500 & 38,816 & 65,26 \\
\hline & Température $\left({ }^{\circ} \mathrm{C}\right)$ & 26,900 & 28,600 & 27,638 & 0,585 & 2,12 \\
\hline & Humidité relative (\%) & 74,000 & 80,000 & 76,750 & 2,435 & 3,17 \\
\hline
\end{tabular}

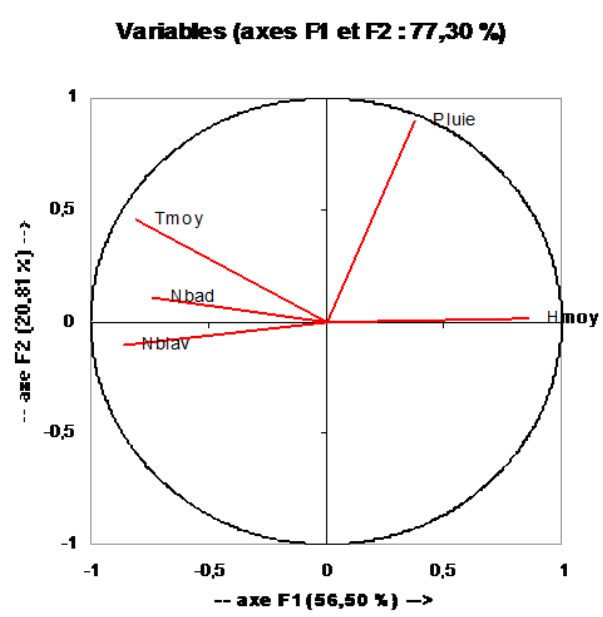

Figure 2 : Structuration des variables étudiées sur les cercles de corrélation du plan 1-2 de 1'ACP dans l'ensemble de l'étude. 
Tableau 2 : Matrice de corrélation entre les 3 paramètres climatiques par date de semis.

\begin{tabular}{|c|c|c|c|c|}
\hline Dates de semis & Variables & Pluviométrie & Température moyenne & Humidité relative \\
\hline \multirow{3}{*}{ Déc. 2000} & Pluviométrie & - & $-0,13$ & $0,78 *$ \\
\hline & Température & & - & $-0,42$ \\
\hline & Humidité relative & & & - \\
\hline \multirow{3}{*}{ Mars 2001} & Pluviométrie & - & 0,04 & $-0,33$ \\
\hline & Température & & - & $-0,75^{*}$ \\
\hline & Humidité relative & & & - \\
\hline \multirow{3}{*}{ Juin 2001} & Pluviométrie & - & $-0,36$ & $-0,12$ \\
\hline & Température & & - & 0,45 \\
\hline & Humidité relative & & & - \\
\hline \multirow{3}{*}{ Août 2001} & Pluviométrie & - & $-0,77^{*}$ & $0,65^{*}$ \\
\hline & Température & & - & $-0,89 *$ \\
\hline & Humidité relative & & & - \\
\hline \multirow{3}{*}{ Oct. 2001} & Pluviométrie & - & $-0,16$ & $-0,07$ \\
\hline & Température & & - & $-0,85^{*}$ \\
\hline & Humidité relative & & & - \\
\hline \multirow{3}{*}{ Février 2002} & Pluviométrie & - & $-0,36$ & $-0,06$ \\
\hline & Température & & - & $-0,82 *$ \\
\hline & Humidité relative & & & - \\
\hline
\end{tabular}

* Valeurs significatives au seuil de $5 \%$

Tableau 3 : Effet de la température moyenne, de la pluviométrie et de l'humidité relative sur les populations de B. tabaci adultes à travers l'analyse de variance des régressions ascendantes simple, double et triple d'ensemble.

\begin{tabular}{llcccccc}
\hline Modèle & Sources & SCE & Ddl & CM & F & P & $\mathbf{R}^{\mathbf{2}}$ (\%) \\
\hline \multirow{3}{*}{1} & Régression & 60,448 & 1 & 60,448 & 20,516 & 0,0001 & 30,80 \\
& Résidu & 135,531 & 46 & 2,946 & & & \\
& Total & 195,979 & 47 & & & & \\
\hline \multirow{2}{*}{2} & Régression & 70,102 & 2 & 35,051 & 12,531 & 0,0001 & 35,80 \\
& Résidu & 125,877 & 45 & 2,797 & & & \\
& Total & 195,979 & 47 & & & & 36,60 \\
3 & Régression & 71,700 & 3 & 23,90 & 8,462 & 0,0001 & \\
& Résidu & 124,279 & 44 & 2,825 & & & \\
& Total & 195,979 & 47 & & & &
\end{tabular}

Légende :

Modèle 1 : Valeurs prédites : (constantes), TMOY

Modèle 2 : Valeurs prédites : (constantes), TMOY, PLUIE

Modèle 3 : Valeurs prédites : (constantes), TMOY, PLUIE, HMOY

Variable dépendante : NBAD

SCE : Somme des carrés des écarts

ddl : nombre de degrés de liberté

$\mathrm{CM}$ : Carré moyen

$\mathrm{F}$ : Rapport du carré moyen de la régression au carré moyen résiduel

$\mathrm{P}$ : Probabilité calculée, significative au seuil de $5 \%$.

$\mathrm{R}^{2}$ : Coefficient de détermination 
Tableau 4 : Effet de la température moyenne, de la pluviométrie et de l'humidité relative sur les populations de larves de $B$. tabaci à travers l'analyse de variance des régressions ascendantes simple, double et triple d'ensemble.

\begin{tabular}{clcccccc}
\hline Modèle & Sources & SCE & Ddl & CM & F & $\mathbf{P}$ & $\mathbf{R}^{\mathbf{2}} \mathbf{( \% )}$ \\
\hline \multirow{2}{*}{1} & Régression & 47,195 & 1 & 47,195 & 20,20 & 0,0001 & 30,50 \\
& Résidu & 107,472 & 46 & 2,336 & & & \\
& Total & 154,667 & 47 & & & & \\
& & & & & & & \\
\multirow{2}{*}{2} & Régression & 66,793 & 2 & 33,396 & 17,102 & 0,0001 & 43,20 \\
& Résidu & 87,874 & 45 & 1,953 & & & \\
& Total & 154,667 & 47 & & & & \\
& & & & & & & \\
& Régression & 79,644 & 3 & 26,548 & 15,570 & 0,0001 & 51,50 \\
& Résidu & 75,022 & 44 & 1,705 & & & \\
& Total & 154,667 & 47 & & & & \\
\hline
\end{tabular}

Légende :

Modèle 1 : Valeurs prédites : (constantes), TMOY

Modèle 2 : Valeurs prédites : (constantes), TMOY, PLUIE

Modèle 3 : Valeurs prédites : (constantes), TMOY, PLUIE, HMOY

Variable dépendante : NBLAV

SCE : Somme des carrés des écarts

ddl : nombre de degrés de liberté

$\mathrm{CM}$ : Carré moyen

F : Rapport du carré moyen de la régression au carré moyen résiduel

$\mathrm{P}$ : Probabilité calculée, significative au seuil de $5 \%$.

$\mathrm{R}^{2}$ : Coefficient de détermination

Tableau 5 : Matrice de corrélation entre les populations de $B$. tabaci et les 3 paramètres climatiques étudiés.

\begin{tabular}{lccccc}
\hline Variables & $\begin{array}{c}\text { Nombre } \\
\text { d'adultes }\end{array}$ & $\begin{array}{c}\text { Nombre } \\
\text { de larves }\end{array}$ & Pluviométrie & $\begin{array}{c}\text { Température } \\
\text { moyenne }\end{array}$ & $\begin{array}{c}\text { Humidité } \\
\text { relative }\end{array}$ \\
\hline Nombre d'adultes & - & $0,55^{*}$ & $-0,20$ & $0,56^{*}$ & $-0,41^{*}$ \\
Nombre de larves & & - & $-0,34^{*}$ & $0,55^{*}$ & $-0,69^{*}$ \\
Pluviométrie & & & - & 0,04 & $0,32^{*}$ \\
Température & & & & - & $-0,70^{*}$ \\
Humidité relative & & & & & -
\end{tabular}

* Valeurs significatives au seuil de $5 \%$

\section{DISCUSSION}

L'influence des paramètres climatiques étudiés sur les populations de la mouche blanche a été significative et a permis de déceler une forte corrélation entre le nombre de larves de l'insecte par feuille, la température moyenne et l'humidité relative, alors que le nombre d'adultes par plant n'était corrélé à aucun paramètre climatique. La température moyenne, la pluviométrie et l'humidité relative pourraient ainsi l'une ou l'autre être prise individuellement pour représenter la variabilité climatique comme l'indique la matrice de corrélation d'ensemble (N'zi, 2010). Cependant, le poids de ces paramètres climatiques n'a pas pu être quantifié 
concernant l'évolution des populations de la mouche blanche avec la corrélation de Pearson. Les effets et la qualité de l'ajustement des données au modèle linéaire choisi représenté par le coefficient de détermination $\mathrm{R}^{2}$ ont permis de le faire. $\mathrm{La}$ contribution des paramètres climatiques individuels dans l'évolution des populations de la mouche blanche a donc été envisagée dans le but de donner de plus amples informations sur les phénomènes étudiés. A cet effet, des équations de droite de régression issues de l'analyse de variance de la régression ont été identifiées. Ces relations entre les paramètres climatiques et les populations de la mouche blanche ne sont donc qu'une indication générale comme l'a mentionné Issali (2008). Une hiérarchie a toutefois été observée entre les 3 paramètres climatiques conformément à l'évolution des populations de la mouche blanche. La température moyenne constitue le paramètre climatique le plus corrélé à la population d'adultes de la mouche blanche (b/TMOY = $0,7608)$. Ce paramètre climatique est encore le plus corrélé à la population de larves de la mouche blanche $(\mathrm{b} / \mathrm{TMOY}=0,6780)$. L'intensité de la liaison existant entre les 2 paramètres corrélatifs au niveau de l'équation de droite associée dépend de la valeur du coefficient de régression (Issali, 2008). La pluviométrie viendrait par conséquent en seconde position pour les adultes et aussi pour les larves de l'insecte, et l'humidité relative en troisième position. Cette hiérarchie suggère que la température moyenne peut préférentiellement être utilisée pour analyser l'influence du climat sur les populations de la mouche blanche. La contribution de la température moyenne, de la pluviométrie et de l'humidité relative à la variation des populations de la mouche blanche a été relativement élevée, mais moins pour les adultes $\left(\mathrm{R}^{2}=35,80 \%\right)$ comparée à celle des larves $\left(\mathrm{R}^{2}=43,20 \%\right)$. La présence de l'insecte a été effective à tous les semis mais à des niveaux variables. La mouche blanche étant un organisme vivant, son développement est subséquent aux variations climatiques surtout de la température et de la pluviométrie (Pavis, 2006 ; Sentis et al., 2012 ; Camara et al., 2016 ; Didi et al., 2018).

Par semis individuel toutefois, la contribution des trois paramètres climatiques dans l'évolution des populations de la mouche blanche n'a pas donné de résultats statistiquement significatifs c'est-à-dire des résultats montrant l'influence ou la contribution de chaque paramètre climatique sur les populations d'adultes par plant et de larves par feuille de l'insecte. En effet, les semis de juin et d'août 2001 ont été les moins sujets à la pullulation de la mouche blanche. Ces résultats confirment les travaux de N'guessan (2001) qui a mentionné qu'aucune relation n'avait été observée entre la température moyenne, la pluviométrie, le niveau d'infestation et les populations d'adultes de B. tabaci sur le gombo en Côte d'Ivoire.

La température moyenne a davantage influencé les populations de larves par feuille et d'adultes par plant de l'insecte, dans l'ensemble. L'évolution de la température moyenne entraînerait donc la prolifération des adultes par plant et des larves par feuille de la mouche blanche. Les températures étant connues pour agir sur le développement de la mouche blanche en serre comme en plein champ (Sentis et al., 2012 ; Brodeur, 2013).

La pluviométrie a eu un impact négatif considérable seulement sur les populations de larves par feuille de l'insecte. L'augmentation de la pluviométrie diminuerait par conséquent les populations de larves de la mouche blanche situées sur la face 
inférieure des feuilles des variétés de tomate cultivées. Ces larves étant généralement immobiles, ou se déplaçant très peu (Mbiyavanga, 2015), sont plus sujettes aux intempéries. Ces résultats confirment ceux obtenus par d'autres auteurs tels qu'Anzola et Lastra (2008) et N'zi et al. (2010) qui ont mentionné que la pluie affectait les effectifs de larves sur la tomate et des adultes de l'insecte sur le cotonnier (Didi et al., 2018). En effet, pendant les semis de décembre 2000 et d'octobre 2001, seules les populations de larves de la mouche blanche ont manifesté une relation négative avec la pluviométrie. Pendant ces périodes, la pluviométrie aurait réduit les effectifs de larves par feuille de l'insecte. Toutefois, l'absence de relation entre les adultes de la mouche blanche et la pluviométrie suggèrerait que la quantité de pluies tombées n'a pas influencé l'effectif d'adultes par plant de la mouche blanche, probablement à cause de leur extrême mobilité comparés aux larves (N'zi, 2010 ; Mbiyavanga, 2015).

L'humidité relative a généralement agi sur le développement des populations de la mouche blanche. Les corrélations observées ont été toutes négatives. Ce qui implique qu'avec l'augmentation de l'humidité ambiante il $y$ aurait une diminution proportionnelle des populations de larves par feuille et d'adultes par plant de la mouche blanche (N'zi, 2010) dans les champs des variétés de tomate cultivées. Ainsi, bien qu'il soit mentionné que divers facteurs tels que l'intensification et la culture successive des plants de tomate, les pratiques culturales variées, etc., puissent avoir une incidence sur l'activité de la mouche blanche, les plus récurrents sont les conditions climatiques (N'zi, 2010 ; Selvaraj et al., 2012) La variabilité climatique qui se manifeste souvent par une modification du régime des précipitations et par une diminution des hauteurs annuelles
(Brou et al., 2005; Selvaraj et al., 2012; Ahoussi et al., 2013), influe considérablement sur les populations de la mouche blanche, démontrant ainsi que le climat joue un rôle primordial dans son développement. De plus, Graff (2006) a mis en évidence la corrélation entre la reprise des activités de la mouche blanche et les conditions climatiques, par observation de la reprise des pontes, durant les jours qui suivent une période pluvieuse ou de forte humidité.

$\mathrm{Au}$ vu des résultats obtenus, entreprendre le calage subséquent de cycles culturaux permettrait d'éviter l'exposition des plants des variétés de tomate à une forte pression de la mouche blanche et de la virose sur la station de recherche.

\section{Conclusion}

Il ressort de cette étude que la présence de la mouche blanche a été effective à tous les semis. Les paramètres climatiques ont permis de prédire les effectifs de la mouche blanche à travers l'analyse de la régression. Dans l'ensemble, ces trois paramètres climatiques ont diversement contribué à l'évolution des effectifs de l'insecte. La température moyenne a été identifiée comme la plus corrélée aux fluctuations de l'insecte, suivie de la pluviométrie et de l'humidité relative. Le calage des périodes de culture de la tomate permettrait d'éviter une forte pullulation de l'insecte. Des relations entre ces trois paramètres climatiques ont été observées et elles ont permis de déterminer que l'un ou l'autre de ces paramètres pouvait servir à décrire le climat pour la lutte contre ce ravageur et la virose qu'il engendre chez la tomate et chez d'autres espèces végétales qui continuent de subir de nos jours les conséquences néfastes de sa prolifération. 


\section{CONFLIT D'INTERETS}

Les auteurs déclarent qu'ils n'ont aucun conflit d'intérêts.

\section{CONTRIBUTIONS DES AUTEURS}

J-CN a assuré la collecte, l'analyse des données, l'élaboration du projet d'article et la correction du manuscrit. CK a participé à l'analyse des données et la correction de l'article. AS-PN a contribué à la rédaction du manuscrit.

\section{PEMERCIEMENTS}

Nous sommes reconnaissants envers la SO.D.EX.AM (Société d'Exploitation Aéroportuaire et Météorologique) pour les données météorologiques et l'AVRDC (Asian Vegetable Research Development Center) pour la mise à disposition des semences de tomate.

\section{REFERENCES}

Ahoussi KE, Koffi YB, Kouassi AM, Soro G, Soro N, Biémi J. 2013. Étude de la variabilité hydroclimatique et de ses conséquences sur les ressources en eau du Sud forestier et agricole de la Côte d'Ivoire : cas de la région d'AbidjanAgboville. Int. J. Pure App. Biosci., 1(6): 30-50.

Anzola D, Lastra R. 2008. Whiteflies Population and its Impact on the Incidence of Tomato Yellow Mosaic Virus in Venezuela. J. Phytopathol., 112(4): 363-366. DOI: $10.1111 /$ j.14390434.1985.tb00815.x

AVRDC (The World Vegetable Center). 2005. Tomato Breeding. Sources of Resistance to Whitefly-transmitted Geminiviruses. AVRDC, Sheet 3, Taiwan, p. 5.

Babu RM, Reddy, Reddy RVSK, Reddy KR, Rani AS, Saidaiah P. 2018. Genetic improvement for yield, quality and leaf curl virus resistance in tomato (Solanum lycopersicum L.). J. Pharmacogn. Phytochem., 1: 1048-1055. www.phytojournal.com.

Brodeur J. 2013. Impact des changements climatiques sur le synchronisme entre les ravageurs et leurs ennemis naturels: conséquences sur la lutte biologique en milieu agricole au Québec. Rapport final, p. 124. www.ouranos.ca/publicationscientifique/RapportBrodeur2013.pdf.

Brou YT, Akindès F, Bigot S. 2005. La variabilité climatique en Côte d'Ivoire: entre perceptions sociales et réponses agricoles. Cah. Agric., 14(6): 533-540.

Camara M, Sarr SM, Sarr I, Noba K. 2016. Caractérisations morphologique et génétique d'espèces d'aleurodes prélevées dans les cultures de tomate atteinte de la virose du TYLC, dans la zone des Niayes au Sénégal. Int. J. Biol. Chem. Sci., 10(4): 1562-1572. DOI: http://dx.doi.org/10.4314/ijbcs.v10i4.9

Didi GJR, Kone PWE, Ochou GEC, Dekoula SC, Kouakou M, Bini KKN, Yao MDS, Mamadou D, Ochou OG. 2018. Évolution spatio-temporelle des infestations de la mouche blanche Bemisia tabaci (Gennadius, 1889) associées à la culture cotonnière en Côte d'Ivoire. J. Appl. Biosc., 121: 1220212210.

DOI: https://dx.doi.org/10.4314/jab.v121i1.10

FAO (Food and Agriculture Organization of the United Nations). 2018. Database results. FAO.

Fondio L. 2005. Contribution à la mise au point d'un itinéraire technique pour la culture du gombo Tomi: Abelmoschus caillei (A. Chev.) Stevels (Malvaceae), dans le centre de la Côte d'Ivoire. Thèse de Doctorat, UFR Biosciences, Université de Cocody, Abidjan, p. 129. 
Fondio L, Djidji AH, N'gbesso MFDP, Koné D. 2013. Evaluation de neuf variétés de tomate (Solanum Lycopersicum L.) par rapport au flétrissement bactérien et à la productivité. Int. J. Biol. Chem. Sci., 7(3): $\quad$ 1078-1086. DOI: http://dx.doi.org/10.4314/ijbcs.v7i3.15

Gamarra H, Carhuapoma P, Kreuze J, Kroschel J. 2016. Pest distribution and risk atlas for Africa. Potential global and regional distribution and abundance of agricultural and horticultural pests and associated biocontrol agents under current and future climates. Lima (Peru). International Potato Center (CIP), 154-168. DOI: 10.4160/978929060476112

Gilberston R, Shetty R. 2006. Application de la biotechnologie pour lutter contre la virose de la tomate en Afrique de l'ouest. Activités de développement. Projet USAID-AVRDC. USAID-Mali, p. 3.

Graff V, Lemmet-Burlat S, Bordat D, TrottinCaudal Y. 2006. Encarsia hispida de Santis, parasitoïde de Bemisia tabaci (Gennadius): Efficacité en serres de production d'hibiscus et poinsettia et quelques éléments de biologie en conditions de laboratoire sur tomate, poivron et concombre. Conférence internationale sur les moyens alternatifs de protection des cultures, Lille, 13-15.

Issali AE. 2008. Analyse de l'influence de quelques paramètres climatiques et génétiques sur l'embryogénèse somatique chez le cacaoyer (Theobroma cacao L.). Thèse de Doctorat Unique, option Génétique et Amélioration des Plantes, UFR Biosciences, Université de Cocody-Abidjan, Abidjan, p. 152.

Kouassi AM, Kouamé KF, Koffi YB, Dje KB, Paturel JE, Oulare S. 2010. Analyse de la variabilité climatique et de ses influences sur les régimes pluviométriques saisonniers en Afrique de l'Ouest: cas du bassin versant du N'zi (Bandama) en Côte d'Ivoire. Cybergeo : Eur. J. Geogr., 513. DOI: $10.4000 /$ cybergeo. 23388 .

Koussoube S, Traore F, Some K, BinsoDabire C, Sanon A. 2018. Perception paysanne des principales contraintes et pratiques culturales en production de patate douce au Burkina Faso. J. Appl. Biosci., 126: 12638-12647. DOI: https://dx.doi.org/10.4314/jab.v126i1.2

Lida H, Kitamura T, Honda KI. 2009. Comparison of egg-hatching rate, survival rate and development time of the immature stage between B-and Qbiotypes of Bemisia tabaci (Gennadius) (Homoptera: Aleyrodidae) on various agricultural crops. Appl. Entomol. Zool., 44(2): 267-273. DOI: 10.1303/aez.2009.267

Mbiyavanga NP. 2015. Possibilité de côntrole de Bemisia tabaci par les extraits d'Azadirachta indica (neem) et deltaméthrine. Université Pédagogique Nationale.

www.memoireonline.com>Sciences.

N'gbesso MFP, Zohouri GP, Fondio L, Djidji

AH, Konate D. 2013. Etude des caractéristiques de croissance et de l'état sanitaire de six variétés améliorées de niébé [Vigna unguiculata (L)] en zone centrale de Côte d'Ivoire. Int. J. Biol. Chem. Sci., 7(2): 457-467. DOI: http://dx.doi.org/10.4314/ijbcs.v7i2.4

N'guessan KP. 2001. Occurrence spread of okra leaf curl virus (OLCD) disease in Côte d'Ivoire. Agron. Afr., 13(1): 35-43.

N'zi JC. 2010. Contribution à la sélection de la tomate [Solanum lycopersicum L. (Solanaceae)] pour la tolérance à l'enroulement jaunissant des feuilles (TYLCD) en Côte d'Ivoire. Mémoire de 
Doctorat Unique, option Génétique et Amélioration des plantes, UFR Biosciences, Université de Cocody, Abidjan, p. 213.

N'zi JC, Kouamé C, Fondio L, Djidji AH, Nguetta ASP, Sangaré A. 2010. Evolution des populations de Bemisia tabaci Genn. Selon les variétés de tomate (Solanum lycopersicum L.) au Centre de la Côte d'Ivoire. Sci. Nat., 7(1): 31-40. DOI:

http://dx.doi.org/10.4314/scinat.v7i1.599 18

Pavis C. 2006. Structure des populations des bioagresseurs. Une grande variabilité spatiale et temporelle des populations des bioagresseurs et d'auxiliaires. INRA, Antilles-Guyane, France. http://www.antilles.inra.fr.

Rubio L, Herrero JR, Sarrio J, Moreno P, Guerri J. 2003. A new approach to evaluate relative resistance and tolerance of tomato cultivars to Begomoviruses causing the tomato yellow leaf curl disease in Spain. Plant Pathol., 52: 763-
$769 . \quad$ DOI: $\quad 10.1111 / \mathrm{j} .1365-$

3059.2003.00926.x

SAS (Statistical Analysis System). 2003. SAS user's guide. SAS Institute, N.C. State University, USA, p. 650.

Sarr M, Badiane D, Sane B. 2016. Evaluation de l'efficacité de nouveaux programmes de protection phytosanitaire contre les principaux ravageurs $\mathrm{du}$ cotonnier Gossypium hirsutum L. au Sénégal. Int. J. Biol. Chem. Sci., 10(5): 2163-2174. DOI:

http://dx.doi.org/10.4314/ijbcs.v10i5.18

Sentis A, Hemptinne JL, Brodeur J. 2012. Using functional response modelling to investigate the effect of temperature on predator feeding and energetic efficiency. Oecologia, 169: 1117-1125. DOI: https://doi.org/10.1007/s00442012-2255-6

Selvaraj S, Ramesh V, 2012. Seasonal abundance of whitefly, Bemisia tabaci Gennadius and their relation to weather parameters in cotton. Int. J. Food. Agric. Vet. Sci., 2(3): 57-63. 